\title{
Antimicrobial susceptibility profiles of porcine mycoplasmas isolated from samples collected in southern Europe
}

\author{
Rubén S. Rosales, Ana S. Ramírez (iD, María M. Tavío, Carlos Poveda and José B. Poveda
}

\begin{abstract}
Background: Mycoplasma (M.) hyopneumoniae, M. hyorhinis and M. hyosynoviae are significant pathogens for the porcine industry worldwide. The aim of the present study was to determine the antimicrobial susceptibility of six key antimicrobials (tylosin, tilmicosin, tylvalosin, lincomycin, tiamulin and valnemulin) routinely used for treating infections caused by these pathogens. Twenty-seven M. hyopneumoniae, 48 M. hyorhinis and 40 M. hyosynoviae field strains isolated from clinical samples from different Southern European countries between 2013 and 2018 using broth microdilution method were evaluated.

Results: Tylvalosin exhibited the highest in vitro activity among the macrolides assayed, with $\mathrm{MIC}_{90}$ values 4 to 5 two-fold dilutions lower than those of tylosin and tilmicosin. The pleuromutilin valnemulin showed one of the highest in vitro activities against the three mycoplasma species. On the contrary, lincomycin exhibited the highest MIC values of the antimicrobials tested.

Conclusions: The data obtained in the present study supports the use of pleuromutilins and macrolides for the control of infections caused by porcine mycoplasmas. The use of lincomycin for the treatment of porcine mycoplasma infections should be carefully evaluated due to the presence of circulating field isolates with decreased susceptibility to this antimicrobial.
\end{abstract}

Keywords: Porcine mycoplasmas, Antimicrobial susceptibility, Macrolides, Lincosamides, Pleuromutilins, MIC

\section{Background}

Mycoplasma (M.) hyopneumoniae, M. hyorhinis and M. hyosynoviae are considered the most relevant Mollicutes to porcine health worldwide, and together with M. suis, a non-culturable haemotropic mycoplasma, represent the main pathogenic mycoplasmas of pigs $[1,2] . M$. hyopneumoniae is a major porcine pathogen, due to its role as the aetiological agent of enzootic pneumonia and also, by interacting with other microorganisms, as a primary pathogen of the porcine respiratory disease complex [3], a disease considered as the most relevant

\footnotetext{
*Correspondence: anasofia.ramirez@ulpgc.es

Unidad de Epidemiología y Medicina Preventiva, Instituto Universitario de Sanidad Animal y Seguridad Alimentaria, Universidad de Las Palmas de Gran Canaria, C/Trasmontana s/n, 35413 Arucas, Gran Canaria, Spain
}

health concern for pig producers [4]. Commercial vaccines are routinely used for the control of this pathogen. However, the analysis of $M$. hyopneumoniae vaccines under field conditions has shown variable efficacy [5], leading in practice to the regular use of antimicrobials against its infections. The antibiotics most frequently used against $M$. hyopneumoniae infections in pigs are aminocyclitols, aminoglycosides, fluoroquinolones, florfenicol, lincosamides, macrolides, pleuromutilins and tetracyclines [6]. On the other hand, M. hyosynoviae is one of the main bacterial pathogen involved in pig lameness [7-9]. Infections caused by this pathogen are characterised by a variable progression, leading in most cases to clinical arthritis. Up to date, there are no commercial vaccines available for the control of this

(c) The Author(s). 2020 Open Access This article is licensed under a Creative Commons Attribution 4.0 International License, which permits use, sharing, adaptation, distribution and reproduction in any medium or format, as long as you give appropriate credit to the original author(s) and the source, provide a link to the Creative Commons licence, and indicate if changes were made. The images or other third party material in this article are included in the article's Creative Commons licence, unless indicated otherwise in a credit line to the material. If material is not included in the article's Creative Commons licence and your intended use is not permitted by statutory regulation or exceeds the permitted use, you will need to obtain permission directly from the copyright holder. To view a copy of this licence, visit http://creativecommons.org/licenses/by/4.0/ The Creative Commons Public Domain Dedication waiver (http://creativecommons.org/publicdomain/zero/1.0/) applies to the data made available in this article, unless otherwise stated in a credit line to the data. 
microorganism, so the control measures rely on farm management and antimicrobial treatment. Enrofloxacin, lincomycin, tetracyclines, tiamulin and tylosin are commonly used for the treatment of $M$. hyosynoviae infections [10]. Furthermore, M. hyorhinis is a ubiquitous porcine pathogen, primarily associated with cases of polyserositis, arthritis and otitis in pigs. In some cases it has been linked to cases of pneumonia, acting as a secondary or opportunistic pathogen. Pneumonia caused by $M$. hyorhinis is clinically indistinguishable from cases produced by M. hyopneumoniae [8-10]. Tetracycline, tiamulin, enrofloxacin, tylosin, tilmicosin and lincomycin have demonstrated in vivo efficacy against this microorganism $[11,12]$. Commercial vaccines are not available for M. hyorhinis, so additional control measures involving improved farm management strategies and the reduction of environmental stressors are required. As described above, the control of porcine mycoplasmosis still depends strongly on the use of antimicrobials. However, the historical overuse of antibiotics in human and animal medicine has led to a current situation of alert, due to the development of resistance that reduces the therapeutic options. In this scenario, the analysis and monitoring of antimicrobial susceptibility has become pivotal in animal health management [13]. The aim of this study was to evaluate the in vitro activity of some of the most relevant antimicrobials used in cases of porcine mycoplasmosis against mycoplasma field strains isolated from Italian, Portuguese and Spanish clinical samples collected between 2013 and 2018.

\section{Results}

\section{Mycoplasma strains}

A total of $27 \mathrm{M}$. hyopneumoniae isolates were obtained from cases of porcine respiratory disease. $M$. hyorhinis isolates (48) were divided into 18 isolates obtained from arthritic joints and 32 from respiratory diseases cases. The $40 \mathrm{M}$. hyosynoviae isolates were obtained from arthritis cases.

\section{MIC values for M. hyopneumoniae}

The MIC range, $\mathrm{MIC}_{50}$ and $\mathrm{MIC}_{90}$ for each antimicrobial tested against $M$. hyopneumoniae are presented in Table 1. For macrolides, isolates tested against tylvalosin showed an MIC range of $0.016-0.06 \mu \mathrm{g} / \mathrm{ml}$ and $\mathrm{MIC}_{50 / 90}$ values of $0.03 / 0.06 \mu \mathrm{g} / \mathrm{ml}$. Results obtained for the other two macrolides tested, tylosin and tilmicosin, were higher than those described for tylvalosin, with an MIC range of $0.06-1$ and $0.25-1 \mu \mathrm{g} / \mathrm{ml}$ respectively and $\mathrm{MIC}_{50 / 90}$ values at $0.5 / 1 \mu \mathrm{g} / \mathrm{ml}$. Lincomycin MIC showed a wide range of dilutions, ranging from 0.06 to $16 \mu \mathrm{g} / \mathrm{ml}$, including the highest MIC value of all M. hyopneumoniae strains tested. $\mathrm{MIC}_{50}$ value found for lincomycin was $0.25 \mu \mathrm{g} / \mathrm{ml}$, while the $\mathrm{MIC}_{90}$ value observed was
$4 \mu \mathrm{g} / \mathrm{ml}$. Tiamulin MIC values were similar to those described for tylosin. For technical issues, valnemulin was only tested against 12 Spanish isolates, and it presented the lowest MIC range $(0.008-0.03 \mu \mathrm{g} / \mathrm{ml})$ of all antimicrobial assayed against $M$. hyopneumoniae. The distribution of MIC values per antimicrobials tested can be found in Table 2. Tilmicosin, tylvalosin and valnemulin showed the narrowest distributions, followed by tylosin and tiamulin.

\section{MIC values for $M$. hyorhinis}

$\mathrm{MIC}_{50}$ and $\mathrm{MIC}_{90}$ for $M$. hyorhinis isolates and distribution of MIC values are summarised in Tables 3 and 4 . MIC range, $\mathrm{MIC}_{50}$ and $\mathrm{MIC}_{90}$ were comparable for tylosin and tilmicosin, with just a 2-fold dilution difference in the lowest $\mathrm{MIC}$ value and $\mathrm{MIC}_{50}$ of tylosin. Tylvalosin showed a reproducible pattern of MIC distribution between the three countries of origin of the isolates, displaying one of the lowest $\mathrm{MIC}_{50} / \mathrm{MIC}_{90}$ of the antimicrobials tested $(0.016 / 0.125 \mu \mathrm{g} / \mathrm{ml})$. MIC values for lincomycin displayed the highest MIC values for all the countries and antimicrobials studied, with an MIC range of $0.125->64$, and the highest $\mathrm{MIC}_{90}$, with a value of $16 \mu \mathrm{g} / \mathrm{ml}$. MIC range for tiamulin was comparable to the range observed for tylosin (MIC range $0.06-8 \mu \mathrm{g} / \mathrm{ml}$ ). Valnemulin MIC values were comparable to tylvalosin for this mycoplasma, apart from $\mathrm{MIC}_{90}$ value, that was the lowest observed for $M$. hyorhinis $(0.03 \mu \mathrm{g} / \mathrm{ml})$. The MIC distribution for all antimicrobials tested showed a broader distribution in comparison to those described for M. hyopneumoniae, with lincomycin presenting the widest distribution observed.

\section{MIC values for M. hyosynoviae}

$\mathrm{MIC}$ ranges, $\mathrm{MIC}_{50}$ and $\mathrm{MIC}_{90}$ for $M$. hyosynoviae were fairly similar between countries, with just one 2-fold dilution difference between MIC values and the geographical origin of the samples (Tables 5 and 6). Valnemulin and tylvalosin displayed the lowest $\mathrm{MIC}_{50} / \mathrm{MIC}_{90}$ for this pathogen $(0.016 / 0.06 \mu \mathrm{g} / \mathrm{ml})$. The highest $\mathrm{MIC}_{50} / \mathrm{MIC}_{90}$ for M. hyosynoviae were those observed for tilmicosin, with concentrations of 1 and $2 \mu \mathrm{g} / \mathrm{ml}$ respectively.

\section{Discussion}

This investigation was aimed at evaluating the antimicrobial susceptibility patterns of selected members of the macrolide, lincosamide and pleuromutilin antimicrobial families against isolates of the most clinically relevant porcine mycoplasmas collected from Southern European countries. Macrolides, lincosamides and pleuromutilins are extremely relevant for porcine health. For instance, the World Organisation for Animal Health (OIE) classifies these antimicrobial families as either critically (macrolides) or highly important antimicrobials 
Table 1 MIC ranges, $\mathrm{MIC}_{50}$ and $\mathrm{MIC}_{90}$ values for 27 Mycoplasma hyopneumoniae isolates. Results are shown in $\mu \mathrm{g} / \mathrm{ml} .{ }^{1} \mathrm{O} n l y ~ 12$ isolates were tested against valnemulin. ${ }^{2} \mathrm{MIC}_{50}$ and $\mathrm{MIC}_{90}$ values were not calculated due to sample size

\begin{tabular}{|c|c|c|c|c|c|c|c|}
\hline & & Tylosin & Tilmicosin & Tylvalosin & Lincomycin & Tiamulin & Valnemulin \\
\hline \multirow[t]{3}{*}{ Spain (22 isolates) $^{1}$} & MIC range & $0.06-1$ & $0.25-1$ & $0.016-0.06$ & $0.06-16$ & $0.06-0.5$ & $0.008-0.03$ \\
\hline & $\mathrm{MIC}_{50}$ & 0.25 & 0.5 & 0.03 & 0.25 & 0.125 & 0.008 \\
\hline & $\mathrm{MIC}_{90}$ & 1 & 1 & 0.06 & 4 & 0.25 & 0.03 \\
\hline Italy (5 isolates) $)^{2}$ & MIC range & $0.25-0.5$ & $0.5-1$ & $0.016-0.06$ & $0.25-1$ & $0.06-0.5$ & - \\
\hline \multirow[t]{3}{*}{ Total (27 isolates) } & MIC range & $0.06-1$ & $0.25-1$ & $0.016-0.06$ & $0.06-16$ & $0.06-0.5$ & - \\
\hline & $\mathrm{MIC}_{50}$ & 0.5 & 0.5 & 0.03 & 0.25 & 0.125 & - \\
\hline & $\mathrm{MIC}_{90}$ & 1 & 1 & 0.06 & 4 & 0.5 & - \\
\hline M. hyopneumoniae strain J & MIC & 0.06 & 0.25 & 0.06 & 0.125 & 0.125 & 0.008 \\
\hline
\end{tabular}

(lincosamides and pleuromutilins) in veterinary medicine [14]. Furthermore, these antimicrobials have remained pivotal for the treatment of infections caused by porcine mycoplasmas in the last decades $[3,12,15,16]$. Due to the limited number of isolates analysed per country, a valid comparison of the MIC values based on their geographical origin could not be achieved.

In our study tylvalosin exhibited the highest in vitro activity among the macrolides assayed against the three mycoplasma species, with $\mathrm{MIC}_{90}$ values 16 to 32 -times lower than those of tylosin and tilmicosin. Similar results were obtained by Tavío et al. [17] after evaluating field strains of M. hyopneumoniae, as well as for the avian mycoplasma M. synoviae and Mycoplasma sp. 1220 [18, 19]. Freely accessible tylvalosin MIC values for mycoplasmas are limited up to date to $M$. hyopneumoniae, $M$. hyorhinis, M. synoviae, Mycoplasma sp. 1220 and M. gallisepticum [17-20]. However, the MIC values observed in our work for this antimicrobial against $M$. hyorhinis and M. hyosynoviae, and the in vitro [17] and in vivo efficacy [21] demonstrated for the treatment of $M$. hyopneumoniae infections, gives prominence to tylvalosin as one of the most effective macrolides against porcine mycoplasmosis, together with tulathromycin, a 15-membered macrolide. Up to date, there are limited comparative data between tylvalosin and tulathromycin MIC values for porcine mycoplasmas. Felde et al. [22] described tulathromycin $\mathrm{MIC}_{90}$ values of 1 and $4 \mu \mathrm{g} / \mathrm{ml}$, 1-4 two-fold dilutions higher to those observed for tylvalosin after analysing Central European M. hyopneumoniae isolates. Conversely, Klein et al. [23] described $\mathrm{MIC}_{90}$ values for tulathromycin of $0.004 \mu \mathrm{g} / \mathrm{ml}$, after analysing 50 European $M$. hyopneumoniae isolates, however, the authors did not include tylvalosin in their analysis. In addition, decreased susceptibility to tylosin and tilmicosin has been previously described in European $M$. hyosynoviae and M. hyopneumoniae isolates $[10,24]$. Although tylvalosin was not evaluated in these studies decreased susceptibility for this antimicrobial has yet to be described. The relatively recent application of tylvalosin for therapy purposes in pigs may explain the absence of decreased susceptibility for this antimicrobial. Also, Andersen and others [25], described the presence of alternative bacterial resistance mechanisms for tylosin in comparison to tylvalosin, which can explain the differences in MIC values between members of the 16membered ring class of macrolides. The tylosin and tilmicosin MIC values were in agreement with those found in M. hyosynoviae [10], M. hyorhinis [12] and $M$. hyopneumoniae $[17,23]$. Based on our data, the macrolides could still be utilised as first-line antimicrobial treatment for the control of mycoplasma disease in pigs. However, evidence about the emergence of macrolideresistant M. hyopneumoniae strains [17, 22, 24, 26],

Table 2 Distribution of 27 Mycoplasma hyopneumoniae isolates based on MIC values. Shaded cells correspond to the MIC ranges of each antimicrobial agent

\begin{tabular}{|c|c|c|c|c|c|c|c|c|c|c|c|c|c|c|c|c|}
\hline \multirow{2}{*}{$\begin{array}{l}\text { Antimicrobial } \\
\text { agent }\end{array}$} & \multicolumn{16}{|c|}{ MIC $(\mu \mathrm{g} / \mathrm{ml})$} \\
\hline & 0.002 & 0.004 & 0.008 & 0.016 & 0.032 & 0.064 & 0.125 & 0.25 & 0.5 & 1 & 2 & 4 & 8 & 16 & 32 & 64 \\
\hline Tylosin & & & & & & 6 & & 7 & 10 & 4 & & & & & & \\
\hline Tilmicosin & & & & & & & & 4 & 11 & 12 & & & & & & \\
\hline Tylvalosin & & & & 10 & 7 & 10 & & & & & & & & & & \\
\hline Lincomycin & & & & & & 6 & 4 & 5 & 5 & 1 & & 3 & 2 & 1 & & \\
\hline Tiamulin & & & & & & 8 & 8 & 6 & 5 & & & & & & & \\
\hline Valnemulin & & & 6 & 3 & 3 & & & & & & & & & & & \\
\hline
\end{tabular}


Table 3 MIC ranges, $\mathrm{MIC}_{50}$ and $\mathrm{MIC}_{90}$ values for 48 Mycoplasma hyorhinis isolates. Results are shown in $\mu \mathrm{g} / \mathrm{ml}$. ${ }^{1}$ Only 19 isolates were tested against valnemulin. ${ }^{2}$ Only 8 isolates were tested against valnemulin. ${ }^{3} \mathrm{~A}$ total of 27 isolates were tested against valnemulin

\begin{tabular}{|c|c|c|c|c|c|c|c|}
\hline & & Tylosin & Tilmicosin & Tylvalosin & Lincomycin & Tiamulin & Valnemulin \\
\hline \multirow[t]{3}{*}{ Spain (25 isolates) $^{1}$} & MIC range & $0.125-0.5$ & $0.25-2$ & $0.016-0.06$ & $0.125-32$ & $0.06-0.5$ & $0.008-1$ \\
\hline & $\mathrm{MIC}_{50}$ & 0.25 & 1 & 0.016 & 0.5 & 0.125 & 0.016 \\
\hline & $\mathrm{MIC}_{90}$ & 0.5 & 1 & 0.03 & 1 & 0.25 & 0.125 \\
\hline \multirow[t]{3}{*}{ Italy (13 isolates) } & MIC range & $0.25-8$ & $0.5-8$ & $0.016-0.5$ & $0.125->64$ & $0.125-8$ & - \\
\hline & $\mathrm{MIC}_{50}$ & 2 & 0.5 & 0.125 & 4 & 0.25 & - \\
\hline & $\mathrm{MIC}_{90}$ & 4 & 4 & 0.5 & 32 & 8 & - \\
\hline \multirow[t]{3}{*}{ Portugal $(10 \text { isolates })^{2}$} & MIC range & $0.125-2$ & $0.5-2$ & $0.016-0.25$ & $0.125-16$ & $0.06-2$ & $0.008-0.03$ \\
\hline & $\mathrm{MIC}_{50}$ & 0.125 & 1 & 0.016 & 0.5 & 0.125 & 0.016 \\
\hline & $\mathrm{MIC}_{90}$ & 1 & 2 & 0.125 & 8 & 2 & 0.016 \\
\hline \multirow[t]{3}{*}{ Total (48 isolates) $)^{3}$} & MIC range & $0.125-8$ & $0.25-8$ & $0.016-0.5$ & $0.125->64$ & $0.06-8$ & $0.008-1$ \\
\hline & $\mathrm{MIC}_{50}$ & 0.25 & 0.5 & 0.016 & 0.5 & 0.125 & 0.016 \\
\hline & $\mathrm{MIC}_{90}$ & 2 & 2 & 0.125 & 16 & 0.5 & 0.03 \\
\hline M. hyorhinis strain BTS-7 & MIC & 0.06 & 0.25 & 0.016 & 0.125 & 0.03 & 0.016 \\
\hline
\end{tabular}

suggests the need for a rational use of this group of antimicrobials in the porcine industry.

With regards to lincomycin, this antimicrobial exhibited the highest MIC values of all the antimicrobial tested, with one strain of $M$. hyorhinis presenting a MIC value of $>64 \mu \mathrm{g} / \mathrm{ml}$. Lincomycin MIC values for $M$. hyopneumoniae displayed a decreased susceptibility pattern in comparison to Spanish isolates [17], evidenced by 32 times higher $\mathrm{MIC}_{90}$ values. However, similar values were observed in previous studies [24, 26]. Current strategies for the control of M. hyopneumoniae include the respiratory exposure of gilts to lung homogenates containing viable strains of this pathogen [27]. However, the presence of circulating strains with decreased susceptibility to certain antimicrobials, as found in our work, or even harbouring multidrug resistance phenotypes [26], requires a careful evaluation of the antimicrobial susceptibility patterns of the strains included in the homogenate prior administration to gilts, in order to reduce the dissemination of resistant isolates of M. hyopneumoniae in the farm. Lincomycin has been previously described as effective in vitro against $M$. hyorhinis [12, 28]. Conversely, our data suggest the presence of resistant strains of $M$. hyorhinis against this antimicrobial in the population studied, in agreement with the data published by Bekö et al. [20] in Hungarian isolates of this pathogen. M. hyorhinis is a common commensal of the upper respiratory tract of pigs, acting as an opportunist pathogen of immunocompromised animal in a variety of clinical presentation [10]. In our study, the population $M$. hyorhinis analysed showed a marked decrease in susceptibility for lincomycin. This antimicrobial has been extensively used as part of infeed medication in porcine farming, administered orally in premix, oral powder and oral solution for years [29]. Therefore, it can be hypothesised that the intensive selective pressure due to in-feed medication for long periods has facilitated the development of a marked decreased susceptibility in our M. hyorhinis strain population. This selective pressure may have enabled in the same way the lincomycin susceptibility and MIC value distribution observed in the $M$. hyosynoviae and $M$.

Table 4 Distribution of 48 Mycoplasma hyorhinis isolates based on MIC values. Shaded cells correspond to the MIC ranges of each antimicrobial agent

\begin{tabular}{|c|c|c|c|c|c|c|c|c|c|c|c|c|c|c|c|c|c|}
\hline \multirow{2}{*}{$\begin{array}{l}\text { Antimicrobial } \\
\text { agent }\end{array}$} & \multicolumn{17}{|c|}{ MIC $(\mu \mathrm{g} / \mathrm{ml})$} \\
\hline & 0.002 & 0.004 & 0.008 & 0.016 & 0.032 & 0.064 & 0.125 & 0.25 & 0.5 & 1 & 2 & 4 & 8 & 16 & 32 & 64 & $>64$ \\
\hline Tylosin & & & & & & & 14 & 14 & 11 & 1 & 3 & 4 & 1 & & & & \\
\hline Tilmicosin & & & & & & & & 6 & 17 & 17 & 4 & 3 & 1 & & & & \\
\hline Tylvalosin & & & & 26 & 7 & 6 & 5 & 2 & 2 & & & & & & & & \\
\hline Lincomycin & & & & & & & 7 & 6 & 19 & 5 & & 1 & 2 & 4 & 3 & & 1 \\
\hline Tiamulin & & & & & & 12 & 20 & 9 & 2 & & 2 & 1 & 2 & & & & \\
\hline Valnemulin & & & 7 & 12 & 5 & & 1 & & & 2 & & & & & & & \\
\hline
\end{tabular}


Table 5 MIC ranges, $\mathrm{MIC}_{50}$ and $\mathrm{MIC}_{90}$ values for 40 Mycoplasma hyosynoviae isolates. Results are shown in $\mu \mathrm{g} / \mathrm{ml} .{ }^{1}$ Only 12 isolates were tested against valnemulin. ${ }^{2}$ Only 2 isolates were tested against valnemulin. ${ }^{3} \mathrm{MIC}_{50}$ and $\mathrm{MIC}_{90}$ values were not calculated due to sample size. ${ }^{4} \mathrm{~A}$ total of 14 isolates were tested against valnemulin

\begin{tabular}{|c|c|c|c|c|c|c|c|}
\hline & & Tylosin & Tilmicosin & Tylvalosin & Lincomycin & Tiamulin & Valnemulin \\
\hline \multirow[t]{3}{*}{$\overline{\text { Spain (18 isolates) }}{ }^{1}$} & MIC range & $0.25-1$ & $0.5-2$ & $0.008-0.06$ & $0.25-1$ & $0.03-0.25$ & $0.016-0.06$ \\
\hline & $\mathrm{MIC}_{50}$ & 0.5 & 1 & 0.016 & 0.25 & 0.06 & 0.016 \\
\hline & $\mathrm{MIC}_{90}$ & 1 & 1 & 0.06 & 0.5 & 0.25 & 0.06 \\
\hline \multirow[t]{3}{*}{ Italy (18 isolates) } & MIC range & $0.5-1$ & $0.5-2$ & $0.008-0.06$ & $0.25-1$ & $0.03-0.5$ & - \\
\hline & $\mathrm{MIC}_{50}$ & 0.5 & 1 & 0.016 & 0.5 & 0.5 & - \\
\hline & $\mathrm{MIC}_{90}$ & 1 & 2 & 0.016 & 1 & 0.5 & - \\
\hline Portugal (4 isolates) $)^{2,3}$ & MIC range & $0.5-1$ & $0.5-1$ & 0.016 & $0.25-1$ & $0.06-0.5$ & $0.016-0.06$ \\
\hline \multirow[t]{3}{*}{ Total $(40 \text { isolates })^{3}$} & MIC range & $0.25-1$ & $0.5-2$ & $0.008-0.06$ & $0.25-1$ & $0.03-0.5$ & $0.016-0.06$ \\
\hline & $\mathrm{MIC}_{50}$ & 0.5 & 1 & 0.016 & 0.5 & 0.125 & 0.016 \\
\hline & $\mathrm{MIC}_{90}$ & 1 & 2 & 0.06 & 1 & 0.5 & 0.06 \\
\hline M. hyosynoviae strain S16 & MIC & 0.25 & 0.25 & 0.016 & 0.125 & 0.03 & 0.008 \\
\hline
\end{tabular}

hyopneumoniae population evaluated. M. hyosynoviae presented similar MIC values to previous reports against lincomycin, with little to no variation observed [10].

Differences in effectivity between pleuromutilins were observed in the three mycoplasma species analysed as previously described [17], with valnemulin exhibiting 8 to 16 times higher activity than tiamulin. MIC values for tiamulin and valnemulin against $M$. hyopneumoniae were in agreement with those previously reported by Tavío et al. [17], and similar to the MIC values observed in isolates from Central Europe, where all isolates were susceptible to a concentration equal or lower than $0.039 \mu \mathrm{g} / \mathrm{ml}$ [22]. However, Klein et al. [23], reported lower MIC values for 50 European isolates of $M$. hyopneumoniae, with a difference in $\mathrm{MIC}_{90}$ found between studies of 8 to 16 times. Also, Hannan et al. [16] described lower MIC values for M. hyopneumoniae and $M$. hyosynoviae against both pleuromutilins. These data suggest a potential decreased activity against pleuromutilins for these two porcine pathogens. M. hyorhinis MIC data presented the broadest range of values for both pleuromutilins tested, in conjunction with the highest MIC values $(8 \mu \mathrm{g} / \mathrm{ml}$ for tiamulin and $1 \mu \mathrm{g} / \mathrm{ml}$ for valnemulin).
Other authors have described lower MIC values for both pleuromutilins in M. hyorhinis for Hungarian isolates [18], with similar results to those described for $M$. hyopneumoniae in the same region after testing the same antimicrobials [22]. Reduced susceptibility to pleuromutilins in mycoplasmas has been linked to mutations in the 23S rRNA gene [30], mutations that also conferred cross-resistance to lincomycin, tilmicosin and tylosin. Even though there are no described mechanisms of pleuromutilin resistance in $M$. hyorhinis, macrolide and lincomycin decreased susceptibility has been associated to multiple mutations in the same gene [31], suggesting a possible cross-resistance phenomenon between pleuromutilins, lincomycin, tilmicosin and tylosin based on the high MIC values for these antimicrobials observed in the M. hyorhinis population studied.

\section{Conclusions}

In conclusion, among the tested antimicrobials, valnemulin and tylvalosin displayed the greatest in vitro activity against the three porcine mycoplasmas species collected from Southern European countries. Although active for M. hyosynoviae, the high MIC values observed

Table 6 Distribution of 40 Mycoplasma hyosynoviae isolates based on MIC values. Shaded cells correspond to the MIC ranges of each antimicrobial agent

\begin{tabular}{|c|c|c|c|c|c|c|c|c|c|c|c|c|c|c|c|c|c|}
\hline \multirow{2}{*}{$\begin{array}{l}\text { Antimicrobial } \\
\text { agent }\end{array}$} & \multicolumn{17}{|c|}{ MIC ( $\mu \mathrm{g} / \mathrm{ml})$} \\
\hline & 0.002 & 0.004 & 0.008 & 0.016 & 0.032 & 0.064 & 0.125 & 0.25 & 0.5 & 1 & 2 & 4 & 8 & 16 & 32 & 64 & $>64$ \\
\hline Tylosin & & & & & & & & 4 & 25 & 11 & & & & & & & \\
\hline Tilmicosin & & & & & & & & & 13 & 20 & 7 & & & & & & \\
\hline Tylvalosin & & & 13 & 20 & & 7 & & & & & & & & & & & \\
\hline Lincomycin & & & & & & & & 13 & 16 & 11 & & & & & & & \\
\hline Tiamulin & & & & & 3 & 10 & 5 & 5 & 17 & & & & & & & & \\
\hline Valnemulin & & & & 7 & & 7 & & & & & & & & & & & \\
\hline
\end{tabular}


for lincomycin against $M$. hyorhinis and M. hyopneumoniae isolates, suggest the need for a more rational approach to the use of this antimicrobial in cases of porcine mycoplasmosis. The molecular basis of the potential cross-resistance for lincomycin, tilmicosin, tylosin and both pleuromutilins in $M$. hyorhinis should be analysed. The differences observed between isolates from different European regions draw special attention to the need for standardised antimicrobial susceptibility testing. Besides, coordinated monitoring schemes for these pathogens in Europe are essentialto effectively tackle the potential emergence of resistant mycoplasma strains in order to maintain an optimal level of health and welfare in the porcine industry.

\section{Methods}

\section{Mycoplasma strains}

Strains were isolated from clinical samples submitted by field veterinarians for routine diagnosis to the mycoplasma diagnostic service of the Instituto Universitario de Sanidad Animal y Seguridad Alimentaria (Gran Canaria, Spain) between 2013 and 2018. Samples were sourced from farrow-to-finish farms and obtained from fattening pigs, gilts and sows with no previous antimicrobial treatment. Clinical samples analysed included nasal swabs from those animals with respiratory disease symptoms compatible with mycoplasmal pneumonia and synovial fluid from cases of lameness.

The isolation of the strains was performed as previously described [32], using modified Friis broth supplemented with equal parts of horse and porcine serum [17]. Pure cultures were obtained as previously described $[32,33]$ and the identity confirmed by biochemical characteristics and specific PCR testing [3, 34]. Strain distribution by species and country of origin can be found in Tables 1,3 and 5 .

The type strains of M. hyopneumoniae (J), M. hyorhinis (BTS-7) and M. hyosynoviae (S16) were used as a positive control. All control strains were sourced from the National Collection of Type Cultures (NCTC).

\section{Antimicrobials}

Tiamulin, tilmicosin, valnemulin and lincomycin were obtained from Fluka Analytical (St Louis, Missouri, USA). Tylosin was obtained from Serva (Heidelberg, Germany) and tylvalosin from Eco Animal Health (London, UK). All antibiotic stock solutions were sterilised by filtration through $0.2 \mu \mathrm{m}$ pore size membrane filters (Millipore).

\section{Minimum inhibitory concentration testing}

Minimum inhibitory concentration (MIC) testing was performed using a microbroth dilution method as previously described [35], using 96-well round base polystyrene microtitre plates (Sarstedt, Nümbrecht, Germany). Antimicrobials were added into the wells following a doubling dilution pattern in order to obtain a final concentration of antibiotic per well that ranged from 0.002 to $64 \mu \mathrm{g} / \mathrm{ml} .100 \mu \mathrm{l}$ of each antimicrobial dilution was added into each well and inoculated with $100 \mu \mathrm{l}$ of a 48 -h mycoplasma culture. The bacterial load present in each inoculum was calculated as described before [17], and adjusted to a final concentration of $10^{5}$ colour-changing units $/ \mathrm{ml}$ of mycoplasma per well. Inoculated plates were then incubated at $37^{\circ} \mathrm{C}$ with constant shaking at $150 \mathrm{rpm}$ in a humidified atmosphere until growth in the drug-free control wells was evident. Bacterial growth was examined daily until a colour change was observed for a maximum of 21 days. MIC testing was performed on three different days, and duplicates of each strain were performed on each of the testing days. MIC was defined as the lowest concentration that completely inhibited growth, shown by a lack of colour change at the time that the drug-free growth control exhibited a colour change, while the negative control remained unchanged. $\mathrm{MIC}_{50}$ and $\mathrm{MIC}_{90}$ are defined as the lowest concentration that completely inhibited growth in 50 and $90 \%$ of the population studied respectively, and MIC ranges were also calculated. Due to the lack of official breakpoints for porcine mycoplasmas, the percentage of resistant isolates was not calculated. MIC values of the type strains can be found in Tables 1,3 and 5. A total of five independent observations per type strain was performed.

\section{Abbrevations}

MIC: Minimum inhibitory concentration; NCTC: National Collection of Type Cultures; OIE: World Organisation for Animal Health; $\mu$ l: Microlitre; $\mu \mathrm{g} /$ $\mathrm{ml}$ : Microgram/millilitre

\section{Acknowledgements \\ Not applicable.}

\section{Authors' contributions}

RSR and ASR cleaned the data, analysed the results and wrote the manuscript. MMT and CP participated in the design of the study, reviewed the results, and reviewed the manuscript. JBP acquired funding, supervised, designed the study, reviewed the results, and reviewed the manuscript. All authors read and approved the final manuscript.

\section{Funding}

The Spanish Ministry of Education (AGL2006-13743/GAN) financed this work. The funders had no role in study design, data collection and analysis, decision to publish, or preparation of the manuscript.

\section{Availability of data and materials}

The datasets used and/or analysed during the current study are available from the corresponding author on reasonable request.

Ethics approval and consent to participate Not applicable.

Consent for publication Not applicable. 


\section{Competing interests}

The authors declare that they have no competing interests.

Received: 4 December 2019 Accepted: 6 August 2020

Published online: 03 September 2020

\section{References}

1. Kobisch M, Friis N. Swine mycoplasmoses. Rev Sci Tech. 1996;15:1569-605.

2. Hoelzle LE, Zeder M, Felder KM, Hoelzle K. Pathobiology of Mycoplasma suis. Vet J. 2014;202:20-5.

3. Maes D, Sibila M, Kuhnert P, Segalés J, Haesebrouck F, Pieters M. Update on Mycoplasma hyopneumoniae infections in pigs: knowledge gaps for improved disease control. Transbound Emerg Dis. 2018:65:110-24.

4. Brockmeier SL, Halbur PG, Thacker EL. Porcine respiratory disease complex. In: Kim A Brogden KA, Guthmiller JM, editors. Polymicrobial Diseases. ASM Press; 2012. https://www.ncbi.nlm.nih.gov/books/NBK2481/.

5. Simionatto S, Marchioro SB, Maes D, Dellagostin OA. Mycoplasma hyopneumoniae: from disease to vaccine development. Vet Microbiol. 2013; 165:234-42.

6. Maes D, Segales J, Meyns T, Sibila M, Pieters M, Haesebrouck F. Control of Mycoplasma hyopneumoniae infections in pigs. Vet Microbiol. 2008;126:297309.

7. Done S, Williamson SM, Strugnell BW. Nervous and Locomotor Systems. In: Zimmerman J, Karriker L, Ramirez A, Schwartz K, Stevenson G, editors. Diseases of Swine. 10th ed. Wiley-Blackwell; 2012. p. 294-328.

8. Roos LR, Surendran Nair M, Rendahl AK, Pieters M. Mycoplasma hyorhinis and Mycoplasma hyosynoviae dual detection patterns in dams and piglets. PLoS One. 2019 3:14:1-12.

9. Pillman D, Surendran Nair M, Schwartz J, Pieters M. Detection of Mycoplasma hyorhinis and Mycoplasma hyosynoviae in oral fluids and correlation with pig lameness scores. Vet Microbiol. 2019 239;108448:1-5.10. Aarestrup F, Friis N. antimicrobial susceptibility testing of Mycoplasma hyosynoviae isolated from pigs during 1968 to 1971 and during 1995 and 1996. Vet Microbiol. 1998;61:33-9.

10. Thacker EL, Minion FC. Mycoplasmosis. In: Zimmerman J, Karriker L, Ramirez A, Schwartz K, Stevenson G, editors. Diseases of Swine. 10th ed. WileyBlackwell; 2012. p. 779-797.

11. Aarestrup FM, Friis NF, Szancer J. Antimicrobial susceptibility of Mycoplasma hyorhinis in a liquid medium compared to a disc assay. Acta Vet Scand. 1998;39:145-7

12. Wu CC, Shryock TR, Lin TL, Faderan M, Veenhuizen MF. Antimicrobial susceptibility of Mycoplasma hyorhinis. Vet Microbiol. 2000;76:25-30.

13. Silley P, Simjee S, Schwarz S. Surveillance and monitoring of antimicrobial resistance and antibiotic consumption in humans and animals. Rev Sci Tech. 2012;31:105-20.

14. Anonymous. OIE List of Antimicrobials of Veterinary Importance. 2007. https://www.oie.int/doc/ged/D9840.PDF. Accessed 25 September 2019.

15. Zimmermann BJ, Ross RF. Determination of sensitivity of Mycoplasma hyosynoviae to tylosin and selected antibacterial drugs by a microtiter technique. Can J Comp Med. 1975;39:17-21.

16. Hannan PCT, Windsor HM, Ripley PH. In vitro susceptibilities of recent field isolates of Mycoplasma hyopneumoniae and Mycoplasma hyosynoviae to valnemulin (Econor ${ }^{\circ}$ ), tiamulin and enrofloxacin and the in vitro development of resistance to certain antimicrobial agents in Mycoplasma hyopneumoniae. Res Vet Sci. 1997;63:157-60.

17. Tavio MM, Poveda C, Assuncao P, Ramírez AS, Poveda JB. In vitro activity of tylvalosin against Spanish field strains of Mycoplasma hyopneumoniae. Vet Rec. 2014;175:539-42.

18. Kreizinger Z, Grózner D, Sulyok KM, Nilsson K, Hrivnák V, Benčina D, et al. Antibiotic susceptibility profiles of Mycoplasma synoviae strains originating from central and Eastern Europe. BMC Vet Res. 2017;13:342-51.

19. Grózner D, Kreizinger Z, Sulyok KM, Rónai Z, Hrivnák V, Turcsányi I, et al. Antibiotic susceptibility profiles of Mycoplasma sp. 1220 strains isolated from geese in Hungary. BMC Vet Res. 2016;12:170-8.

20. Bekő K, Felde O, Sulyok KM, Kreizinger Z, Hrivnák V, Kiss K, et al. Antibiotic susceptibility profiles of mycoplasma hyorhinis strains isolated from swine in Hungary. Vet Microbiol. 2019;228:196-201.

21. Pallarés FJ, Lasa C, Roozen M, Ramis G. Use of tylvalosin in the control of porcine enzootic pneumonia. Vet Rec Open. 2015;2:e000079. https://doi.org/ 10.1136/vetreco-2014-000079 eCollection 2015.
22. Felde O, Kreizinger Z, Sulyok KM, Hrivnák V, Kiss K, Jerzsele Á, et al. Antibiotic susceptibility testing of mycoplasma hyopneumoniae field isolates from Central Europe for fifteen antibiotics by microbroth dilution method. PLoS One. 2018;13(12):e0209030. https://doi.org/10.1371/journal. pone. 0209030

23. Klein U, de Jong A, Moyaert H, El Garch F, Leon R, Richard-Mazet A, et al. Antimicrobial susceptibility monitoring of Mycoplasma hyopneumoniae and Mycoplasma bovis isolated in Europe. Vet Microbiol. 2017;204:188-93.

24. Vicca J, Stakenborg T, Maes D, Butaye P, Peeters J, de Kruif A, et al. In vitro susceptibilities of Mycoplasma hyopneumoniae field isolates. Antimicrob Agents Chemother. 2004:48:4470-2.

25. Andersen NM, Poehlsgaard J, Warrass R, Douthwaite S. Inhibition of protein synthesis on the ribosome by tildipirosin compared with other veterinary macrolides. Antimicrob Agents Chemother. 2012;56:6033-6.

26. Thongkamkoon P, Narongsak W, Kobayashi H, Pathanasophon P, Kishima M, Yamamoto K. In vitro susceptibility of Mycoplasma hyopneumoniae field isolates and occurrence of Fluoroquinolone, macrolides and Lincomycin resistance. J Vet Med Sci. 2013;75:1067-70.

27. Robbins RC, Betlach AM, Mondragon-Evans MR, Pieters M. Development of a herd-specific lung homogenate for exposure to Mycoplasma hyopneumoniae under field conditions. J Swine Health Prod. 2019;27:221-7.

28. Kobayashi H, Sonmez N, Morozumi T, Mitani K, Ito N, Shiono H. In vitro susceptibility of Mycoplasma hyosynoviae and M. hyorhinis to antimicrobial agents. J Vet Med Sci. 1996;58:1107-11.

29. Pyörälä S, Baptiste KE, Catry B, van Duijkeren E, Greko C, Moreno MA. Macrolides and lincosamides in cattle and pigs: use and development of antimicrobial resistance. Vet J. 2014;200:230-9.

30. Li BB, Shen JZ, Cao XY, Wang Y, Dai L, Huang SY, et al. Mutations in $23 S$ rRNA gene associated with decreased susceptibility to tiamulin and valnemulin in Mycoplasma gallisepticum. FEMS Microbiol Lett. 2010;308:144-9.

31. Kobayashi H, Nakajima H, Shimizu Y, et al. Macrolides and Lincomycin susceptibility of Mycoplasma hyorhinis and variable mutation of domain II and V in 23 S ribosomal RNA. J Vet Med Sci. 2005;67:795-800.

32. Assunção P, De la Fe C, Kokotovic B, González O, Poveda JB. The occurrence of mycoplasmas in the lungs of swine in gran Canaria (Spain). Vet Res Commun. 2005;29:453-62.

33. Friis NF. Some recommendations concerning primary isolation of Mycoplasma suipneumoniae and Mycoplasma flocculare a survey. Nord Vet Med. 1975;27:337-9.

34. Marois C, Dory D, Fablet C, Madec F, Kobisch M. Development of a quantitative real-time TaqMan PCR assay for determination of the minimal dose of Mycoplasma hyopneumoniae strain 116 required to induce pneumonia in SPF pigs. J Appl Microbiol. 2010;108:1523-33.

35. Hannan PCT. Guidelines and recommendations for antimicrobial minimum inhibitory concentration (MIC) testing against veterinary mycoplasma species. Vet Res. 2000;31:373-95.

\section{Publisher's Note}

Springer Nature remains neutral with regard to jurisdictional claims in published maps and institutional affiliations.

Ready to submit your research? Choose BMC and benefit from:

- fast, convenient online submission

- thorough peer review by experienced researchers in your field

- rapid publication on acceptance

- support for research data, including large and complex data types

- gold Open Access which fosters wider collaboration and increased citations

- maximum visibility for your research: over $100 \mathrm{M}$ website views per year

At $\mathrm{BMC}$, research is always in progress.

Learn more biomedcentral.com/submissions 